Supporting information for

\title{
Comparison of sulfur cathode reactions between concentrated liquid electrolyte system and solid-state electrolyte system by soft $X$-ray absorption spectroscopy
}

Yao Xiao ${ }^{a}$, Kentaro Yamamoto ${ }^{a,}{ }^{*}$, Yukiko Matsui ${ }^{b}$, Toshiki Watanabe ${ }^{a}$, Atsushi Sakuda ${ }^{c}$, Koji Nakanishi $^{a}$, Tomoki Uchiyama ${ }^{a}$, Akitoshi Hayashic, Shoso Shingubara ${ }^{d}$, Masahiro Tatsumisago ${ }^{c}$, Masashi Ishikawab, Masayoshi Watanabe and Yoshiharu Uchimoto ${ }^{a}$

a. Graduate School of Human and Environmental Studies, Kyoto University, Yoshida-nihonmatsucho, Sakyoku, Kyoto 606-8501, Japan

b. Department of Chemistry and Materials Engineering, Kansai University, 3-3-35 Yamate-cho, Suita, Osaka 564-8680, Japan

c. Graduate School of Engineering, Osaka Prefecture University, Sakai, Osaka 599-8531, Japan

d. Department of Mechanical Engineering, Kansai University, 3-3-35 Yamate-cho, Suita, Osaka 564-8680, Japan

e. Institute of Advanced Sciences, Yokohama National University, 79-5 Tokiwadai, Hodogaya-ku, Yokohama 240-8501, Japan

Corresponding Author

* Kentaro Yamamoto. e-mail: yamamoto.kentaro.4e@kyoto-u.ac.jp 


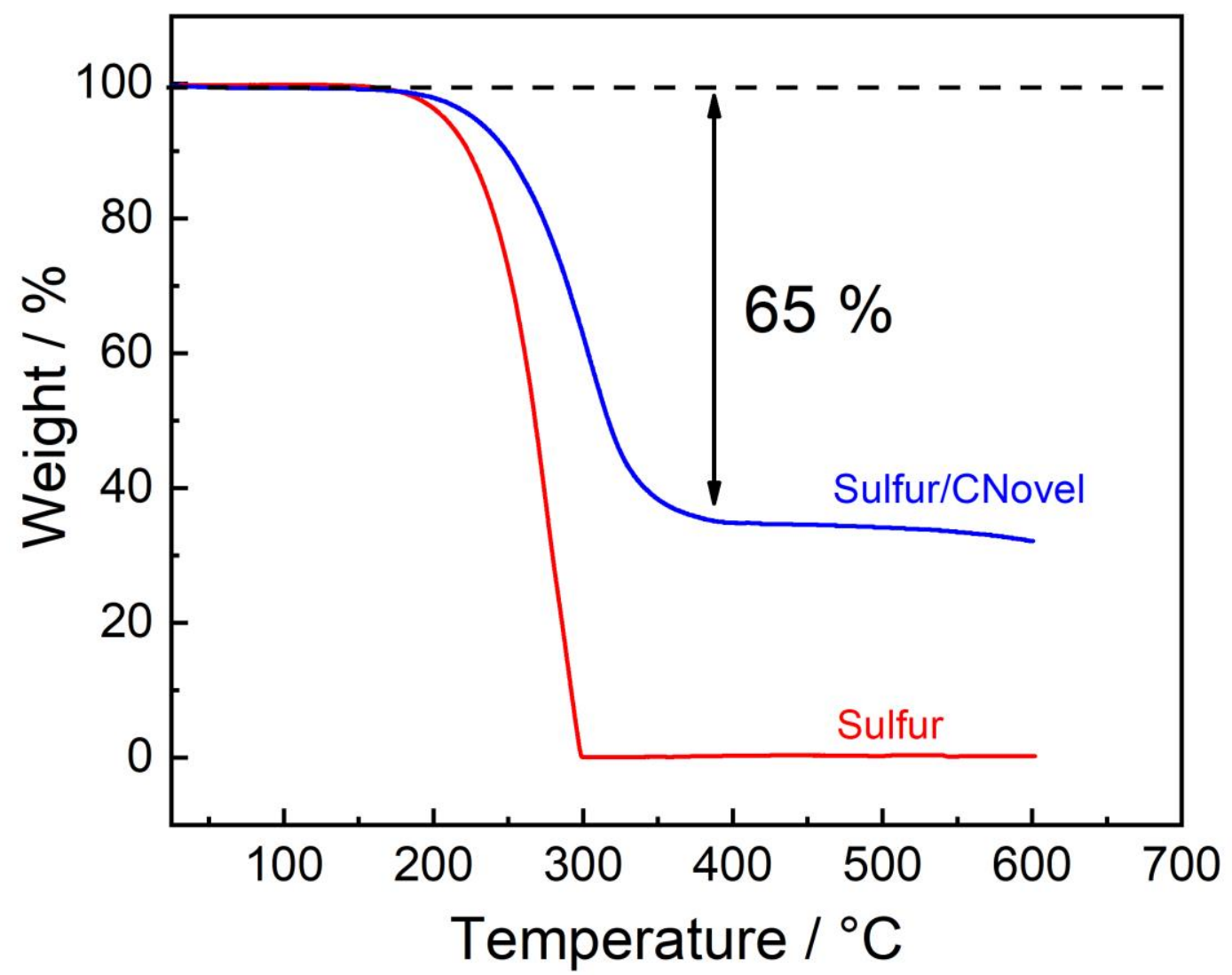

Figure S1. Thermo gravimetric analysis curves of sulfur and sulfur/CNovel powder. 


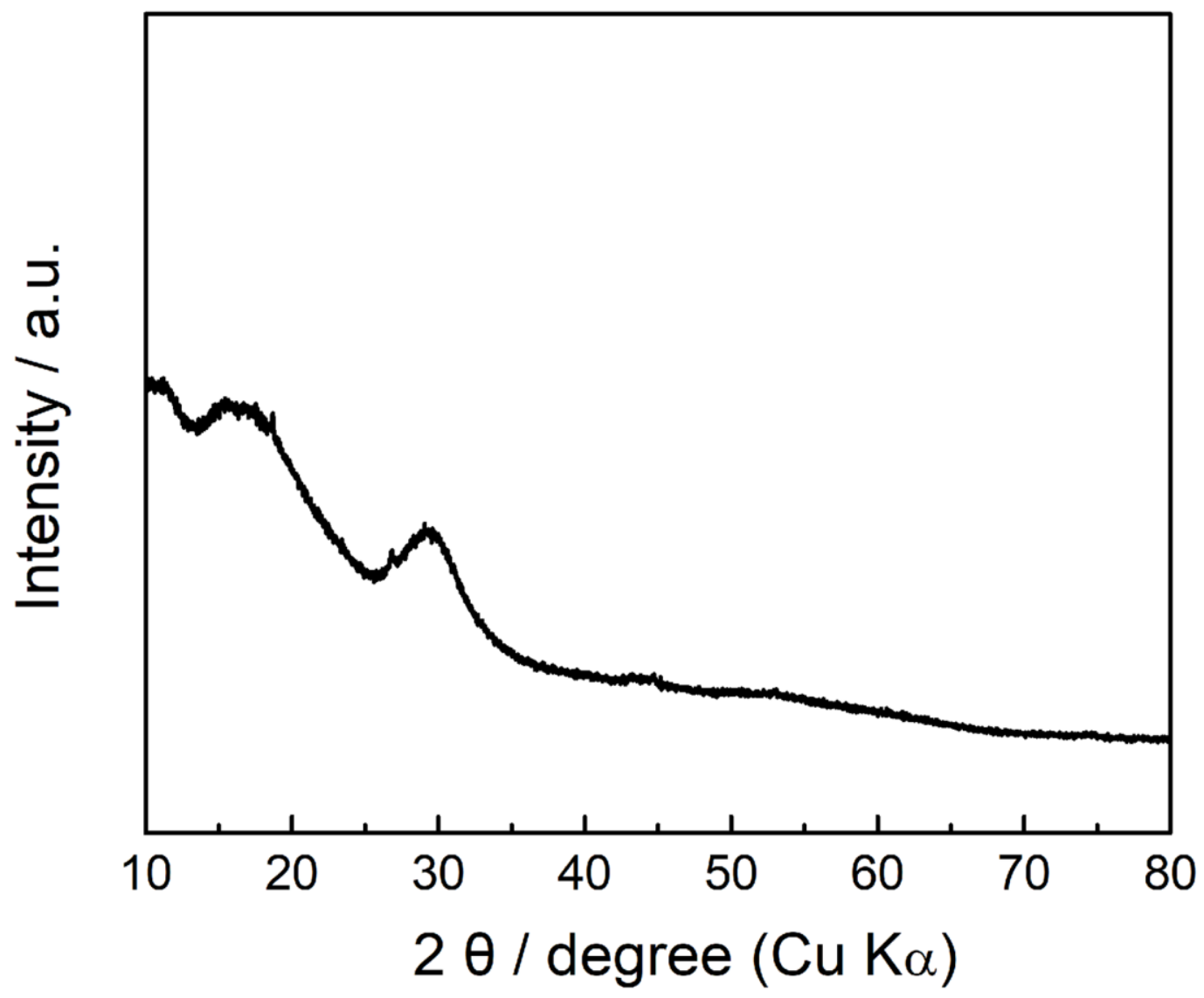

Figure S2. XRD pattern of the prepared $\mathrm{Li}_{3} \mathrm{PS}_{4}$. 
(a)

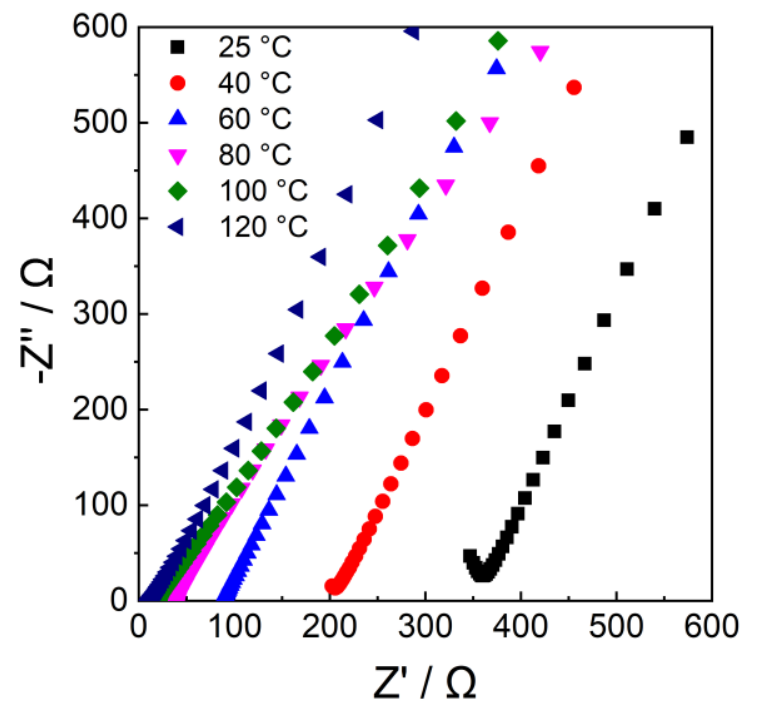

(b)

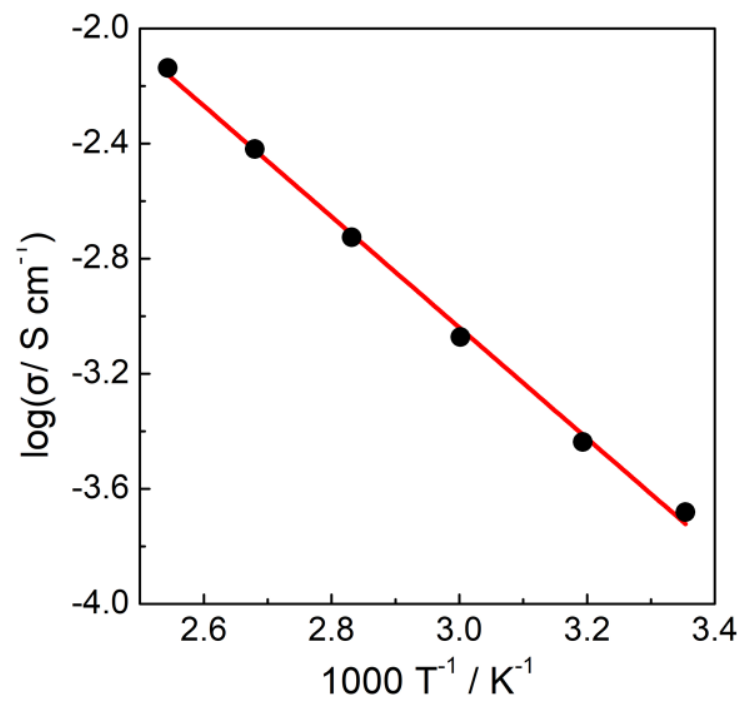

Figure S3. (a) Nyquist plots of the prepared $\mathrm{Li}_{3} \mathrm{PS}_{4}$ at different temperature, (b) Temperature dependence of the ionic conductivity of $\mathrm{Li}_{3} \mathrm{PS}_{4}$. 
(a)
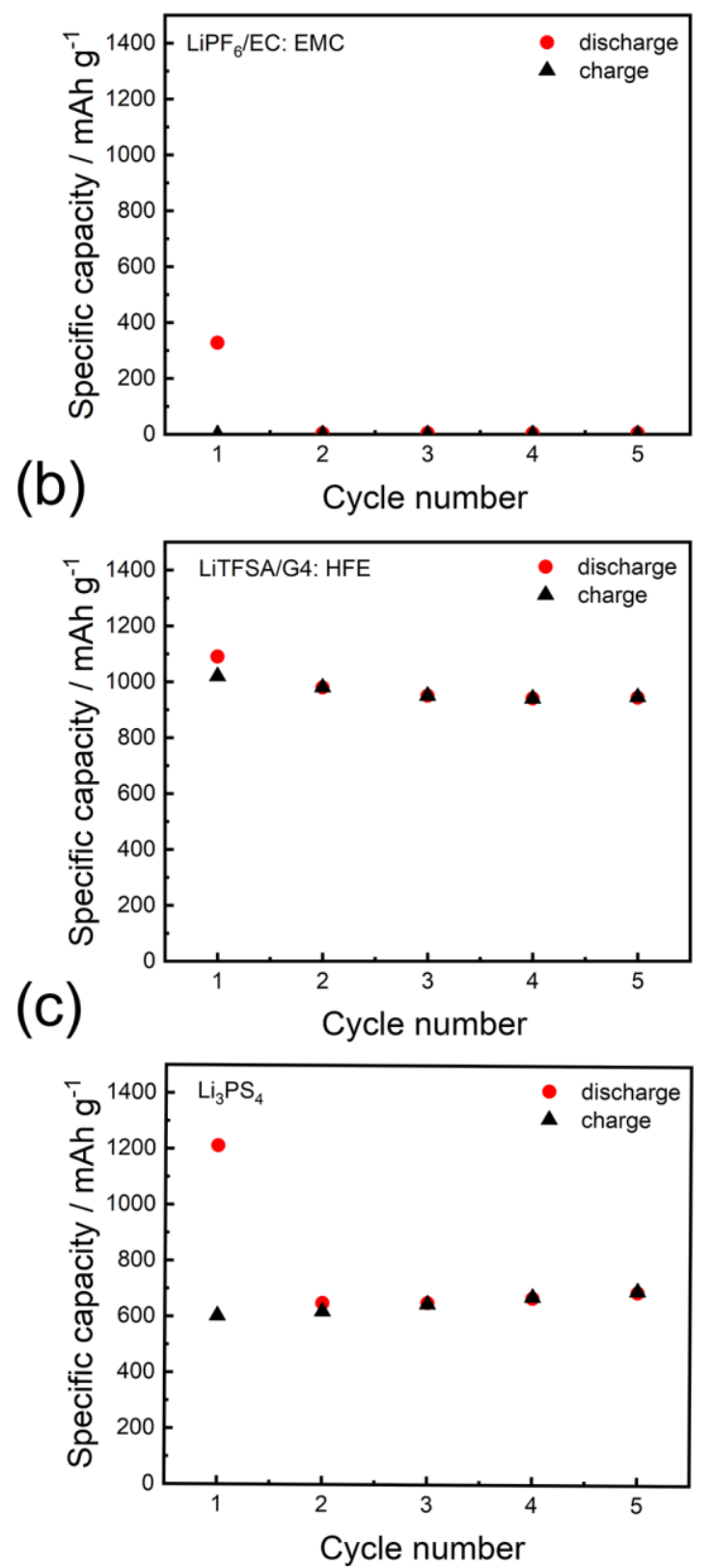

Figure S4. Cyclability for sulfur/CNovel cathode at (a) in theLiPF $6 / E C: E M C$ conventional liquid electrolyte, (b) in the LiTFSA/G4: HFE concentrated liquid electrolyte, and (c) in the $\mathrm{Li}_{3} \mathrm{PS}_{4}$ Solidstate electrolyte. 


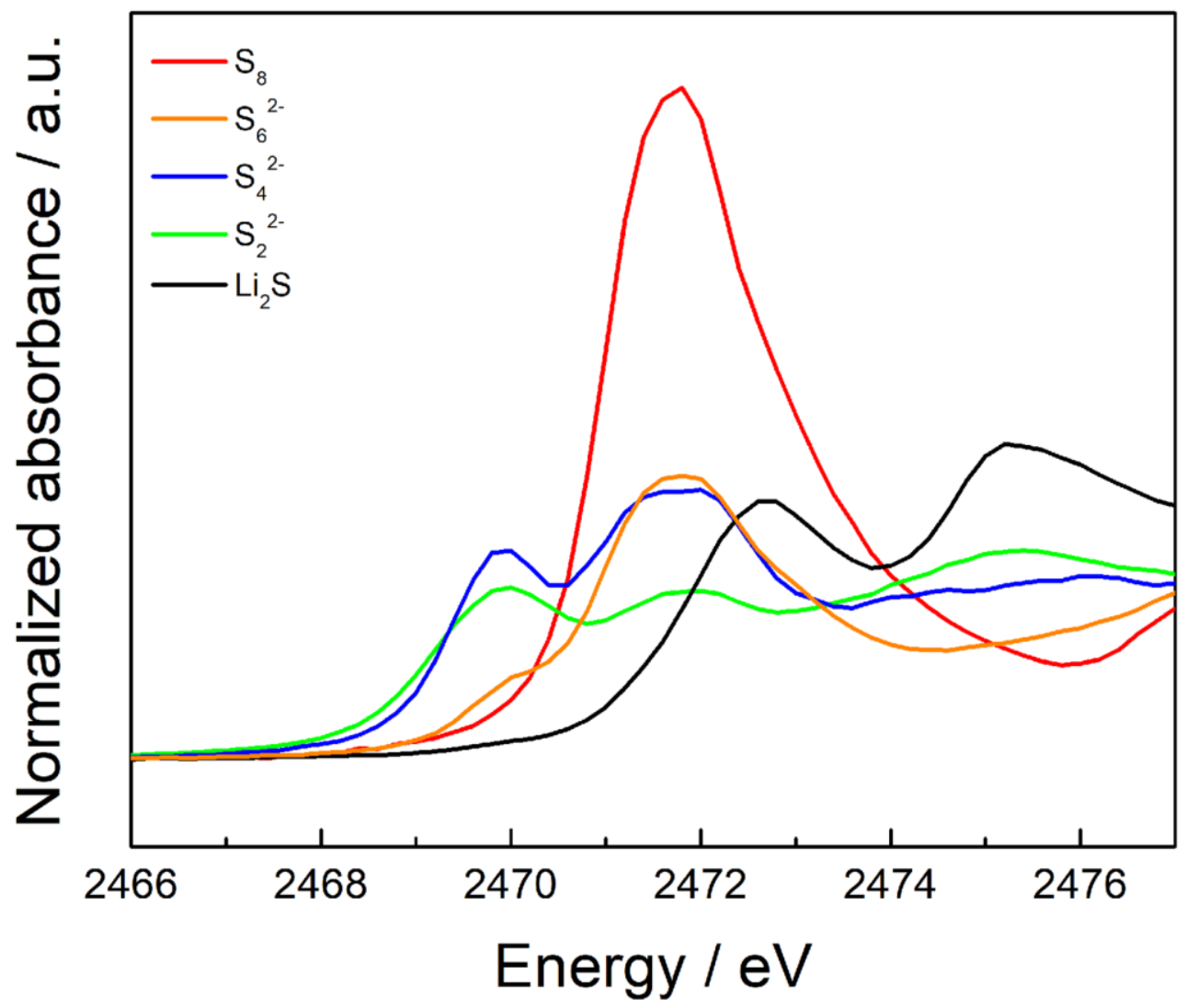

Figure S5. Sulfur $K$-edge XANES of standard materials $\mathrm{S}_{8}, \mathrm{~S}_{6}{ }^{2-}, \mathrm{S}_{4}{ }^{2-}, \mathrm{S}_{2}{ }^{2-}$ and $\mathrm{Li}_{2} \mathrm{~S}$. 


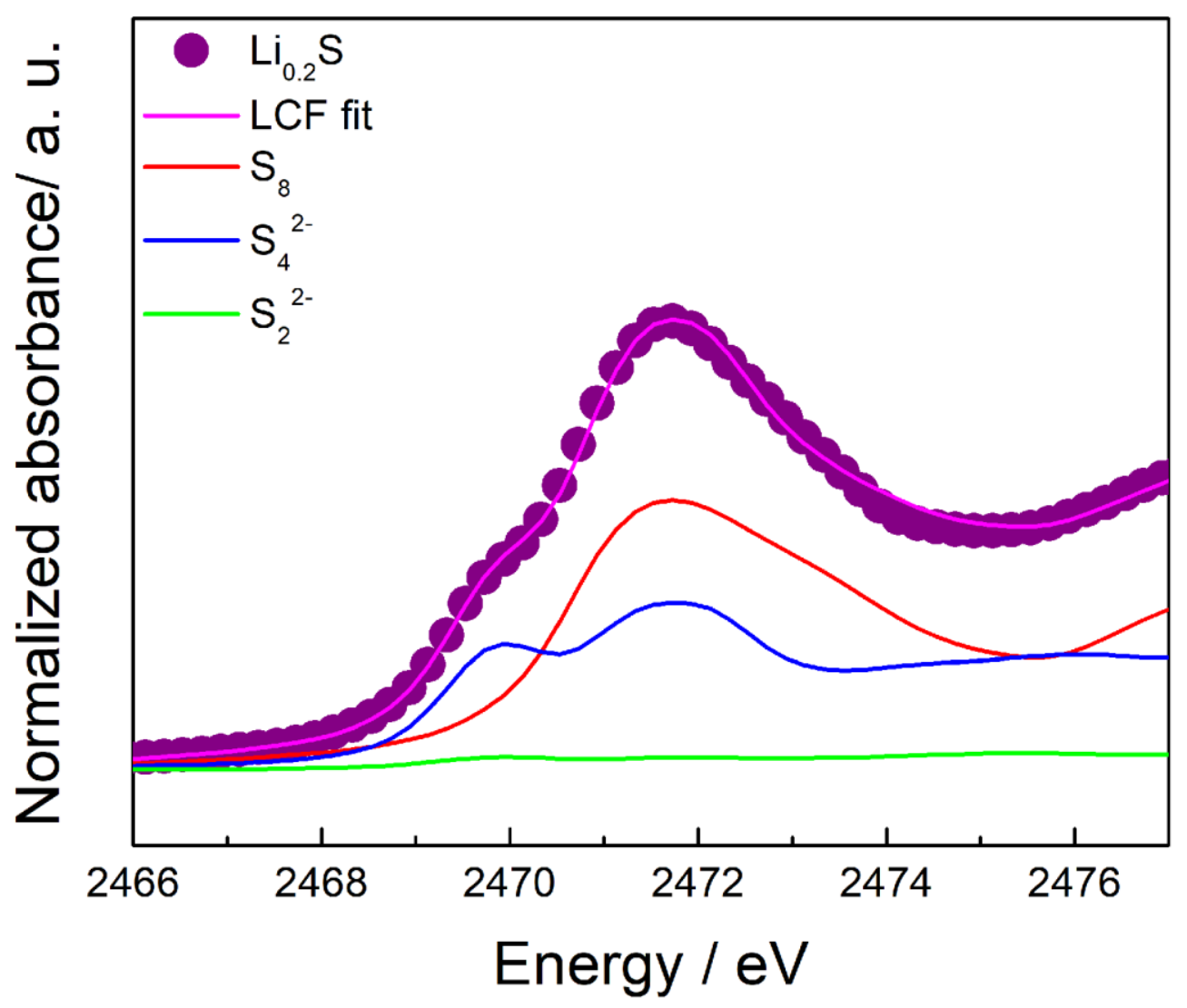

Figure S6. Linear combination fitting result of sulfur $K$-edge XANES of sulfur/CNovel cathode in LiTFSA/G4: HFE concentrated liquid electrolyte at discharge state of $\mathrm{Li}_{0.2} \mathrm{~S}$. 


\section{Linear combination fitting for the sulfur cathode in $\mathrm{Li}_{3} \mathrm{PS}_{4}$ solid electrolyte.}

In the $\mathrm{Li}_{3} \mathrm{PS}_{4}$ solid electrolyte, $\mathrm{S}_{8}, \mathrm{~S}_{6}{ }^{2-}, \mathrm{S}_{4}{ }^{2-}, \mathrm{S}_{2}{ }^{2-}$ and $\mathrm{Li}_{2} \mathrm{~S}$ were used as the stander materials to do the linear combination fitting at first. However, the fitting result can't match well with the XANES spectra data especially for $\mathrm{Li}_{1.0} \mathrm{~S}$ and $\mathrm{Li}_{1.2} \mathrm{~S}$ (Figure $\mathrm{S} 7$ ). The absorption peak at $2471 \mathrm{eV}$ may correspond to $\mathrm{S} K$-edge of the $\mathrm{Li}_{3} \mathrm{PS}_{4}$ (ionic conductor in cathode). At this point, $\mathrm{Li}_{3} \mathrm{PS}_{4}$ was also used as stander material to do linear combination fitting of XANES spectra of each discharge status. As showed in Figure S8, the LCF result can fit well with the XANES spectra of $\mathrm{Li}_{1.0} \mathrm{~S}$ and $\mathrm{Li}_{1.2} \mathrm{~S}$, which means this method can be employed to analysis the polysulfides species changes in all-solid-state battery. 
(a)

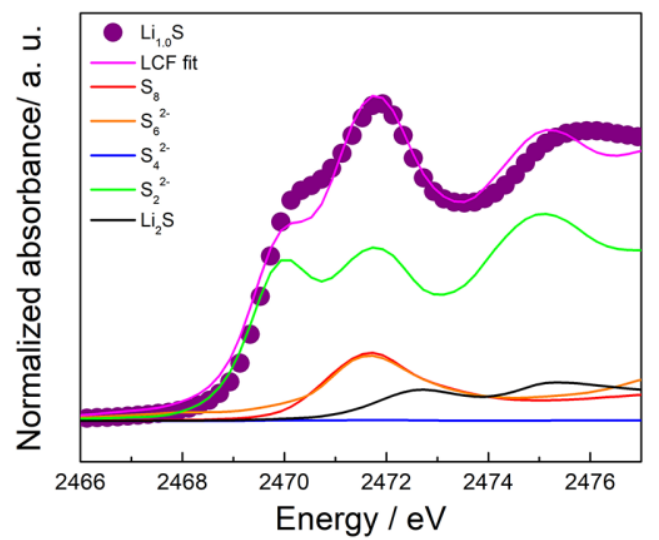

(b)

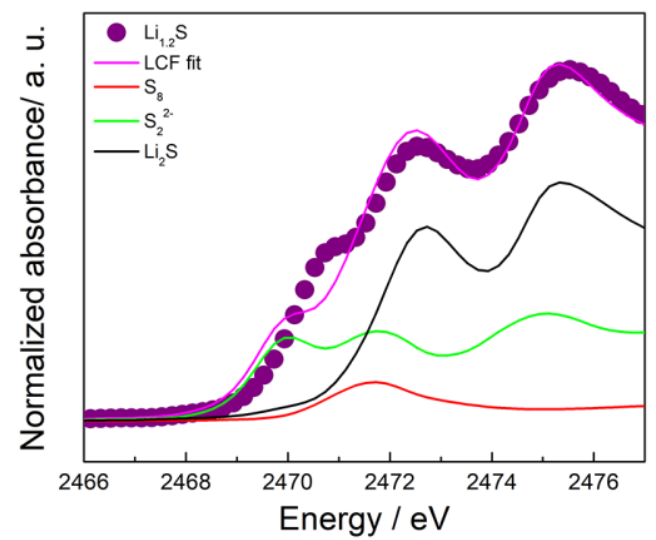

Figure S7. Linear combination fitting result of sulfur $K$-edge XANES of sulfur/CNovel cathode in all solid-state lithium sulfur battery by using stander material without $\mathrm{Li}_{3} \mathrm{PS}_{4}$ at discharge state of (a) $\mathrm{Li}_{1.0} \mathrm{~S}$ and (b) $\mathrm{Li}_{1.0} \mathrm{~S}$. 
(a)

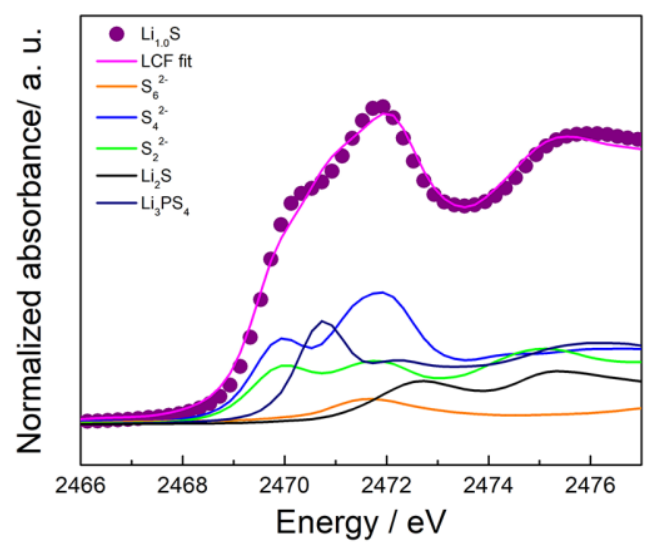

(b)

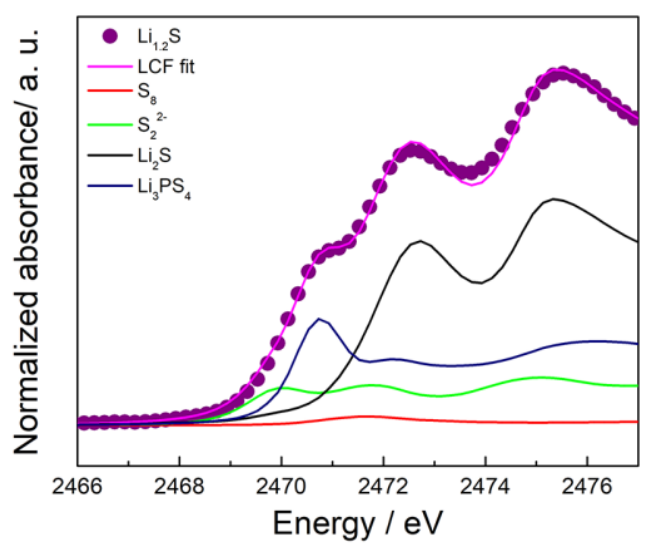

Figure S8. Linear combination fitting result of sulfur $K$-edge XANES of sulfur/CNovel cathode in all solid-state lithium sulfur battery by using stander material with $\mathrm{Li}_{3} \mathrm{PS}_{4}$ at discharge state of (a) $\mathrm{Li}_{1.0} \mathrm{~S}$ and (b) $\mathrm{Li}_{1.2} \mathrm{~S}$. 
(a)

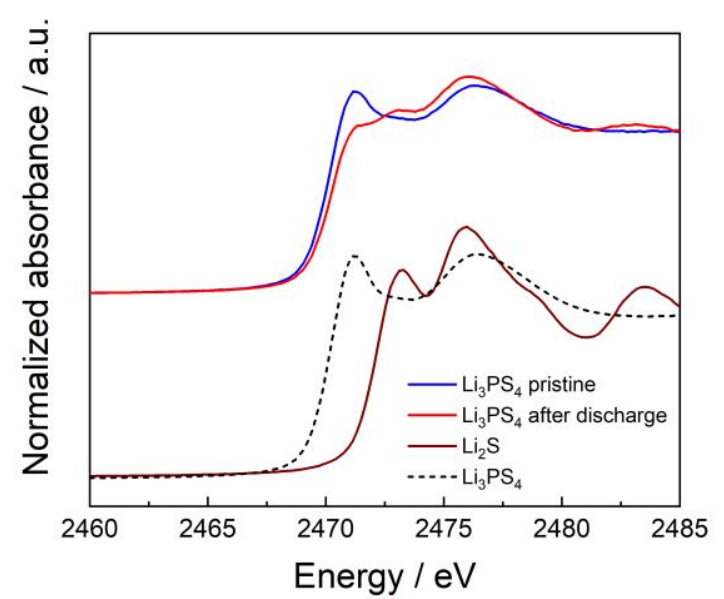

(b)

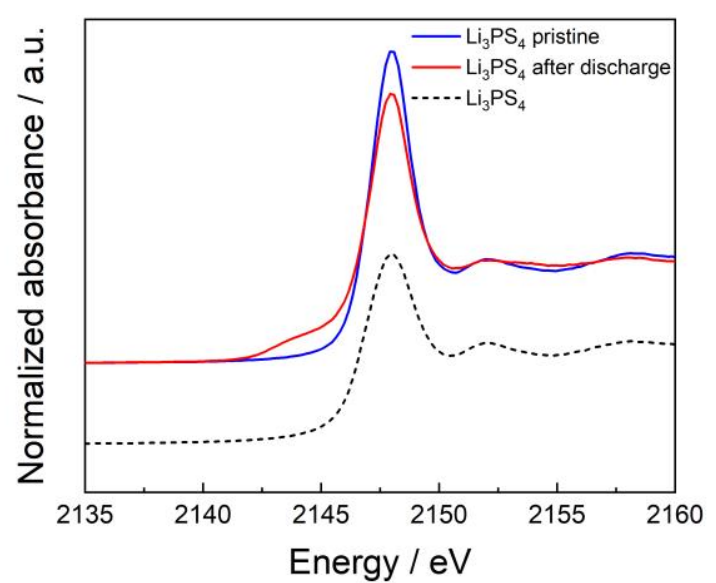

Figure S9. X-ray Absorption Near Edge Structure (XANES) for (a) S K-edge and (b) P K-edge of the $\mathrm{Li}_{3} \mathrm{PS}_{4}$ electrolyte after kept at the potential of $0 \mathrm{~V}\left(\mathrm{vs} . \mathrm{Li}^{+} / \mathrm{Li}\right)$. The XANES spectra of the $\mathrm{Li}_{3} \mathrm{PS}_{4}$ sample before and after electrochemical measurement were collected with partial fluorescence mode at BL13 in Rits SR, Japan. 